\title{
P 004 LIVING IN THE SHADOW OF CANCER: THE EXPERIENCE OF FIRST DEGREE RELATIVES OF PATIENTS WITH ADVANCED COLORECTAL CANCER
}

Nicola Baker, ${ }^{1}$ Angus Dawson, ${ }^{2}$ Sabi Redwood, ${ }^{2}$ Kathy Armour, ${ }^{1}$ Chantal Meystre ${ }^{1}$. ${ }^{1}$ Marie Curie Hospice West Midlands, Solihull, UK; ${ }^{2}$ School of Health and Population Sciences, University of Birmingham, Birmingham, UK

\subsection{6/bmjspcare-2014-000654.44}

Background It is widely accepted that a diagnosis of cancer affects the entire family. However, research has centred on primary caregivers, and little is known about the impact on the wider family. The literature has concentrated on female relatives of breast cancer patients. Studies involving relatives of colorectal cancer patients have focused on their attitudes to screening, or behavioural interventions, and have not attempted to identify their experiences, beliefs or needs.

Aims To gain an in depth understanding of the experiences, beliefs and needs of first-degree relatives of patients diagnosed with advanced colorectal cancer.

Methods Semi-structured interviews were conducted with adult children or siblings of patients with advanced colorectal cancer, referred to a palliative care service. Interviews explored their experience of a cancer diagnosis in the family and their beliefs about cancer. Data was transcribed verbatim and analysed qualitatively using a constant comparison method.

Results 5 categories emerged from the data;

- 1) Emotional burden - The impact of witnessing a loved one with progressive disease was enormous. Relatives described the "shadow" cancer cast over the whole family, and feelings of fear on multiple levels. Emotional challenges were even greater when needing to support several generations within the family.

- 2) Practical burden, which was high even if they did not have a caring role, or live locally.

- 3) Changing family roles accompanying the anticipated death of their relative.

- 4) Feeling "out of the loop" and a need for more information.

- 5) Awareness of own risk - Some relatives described an increased sense of their personal risk of cancer, which exacerbated their fears and represented a significant barrier to open communication in the family.

Conclusion This study highlights the profound impact of advanced colorectal cancer on the wider family, and suggests a 
role for palliative care in identifying and supporting their unmet needs. 